\title{
Defining the Features of Registry Based Randomised Controlled Trials (rRCT): A Systematic Review
}

Niamh 0 Shea ( $\square$ n.oshea@ucc.ie )

University College Cork National University of Ireland https://orcid.org/0000-0002-7684-7588

Joseph Eustace

University College Cork National University of Ireland

Frances Shiely

University College Cork National University of Ireland

\section{Protocol}

Keywords: Registry Based Randomised Controlled Trial, Systematic Review, Protocol, Defining rRCTs

Posted Date: September 9th, 2020

DOI: https://doi.org/10.21203/rs.3.rs-58883/v1

License: (c) (i) This work is licensed under a Creative Commons Attribution 4.0 International License.

Read Full License 


\section{Abstract}

Background: Registry Based Randomised Controlled Trials have been described as pragmatic studies utilising patient data embedded in large scale registries, to facilitate key clinical trial procedures such as case report completion, randomisation and follow up data. While the practice of utilising registries to support the conduct of randomised trials is increasing, the reporting of how a registry is used within a trial can vary, causing difficulty in identifying registry based randomised trials and interpreting their exact definition.

Methods: The databases PubMed, Embase, Cinahl Plus, Scopus and the Cochrane Central Register of Controlled Trials will be searched using a combination of subject headings, MeSH and free text terms. Search terms will be adapted accordingly for each database, with English language articles included and no other filters applied. Also, grey literature and reference lists will be searched, contacting trial authors for clarification when necessary. Two independent reviewers will complete study screening, selection and quality assessment. A preliminary synthesis will be conducted tabulating the relevant evidence into separate data extraction tables. A narrative synthesis approach will be adopted based on the Guidance on the Conduct of Narrative Synthesis in Systematic Reviews.

Results: The present study will synthesise existing registry based randomised trial literature and define their key features.

Conclusions: It is essential that trialists and researchers can review published trials and endeavour to duplicate trial designs. There is a lack of consensus in terms of the reporting of registry based randomised trials, making replication of this emerging trial design difficult. This review will clearly summarise and define the key features of these randomised trials, to allow researchers understand and reproduce the novel registry based randomised controlled trial methodology.

\section{Systematic Review Registration: PROPSERO CRD42020192419}

\section{Background}

While the traditional randomised controlled trial design (RCT) remains gold standard within clinical research for testing the efficacy of new treatments and improving clinical care (1), RCTs are a complex and costly undertaking, with partial external validity and limited follow up of trial participants (2). The last decade has seen an exponential increase in the number of registry-based randomised controlled trials (rRCTs) conducted (3-6). rRCTs have been described as pragmatic trials utilising patient data embedded in large scale, national, registries to facilitate case report form completion, randomisation of trial participants and the acquisition of follow up trial data $(7,8)$. The reported advantages of rRCTs include reduced trial costs, speedy consecutive participant enrolment, completeness of follow-up trial data and trial time saving efficiency (8). 
While various descriptions of the term registry or register exist, the term 'patient registry' is more often used in the field of healthcare (9). This protocol defines the term registry as "an organized system that uses observational study methods to collect uniform data (clinical and other) to evaluate specified outcomes for a population defined by a particular disease, condition, or exposure, and that serves one or more predetermined scientific, clinical, or policy purposes" (10). Registries play a vital role in clinical research as the information collected and stored within these vast databases provide an ideal platform for the conduct of large clinical trials (11).

There have been various examples of rRCTs described in the literature in recent years. The TASTE trial (12), investigated the use of thrombus aspiration in ST-segment elevation in myocardial infarction. Taste Trial researchers devised the phrase "randomized clinical registry trial" to describe their novel application of the Swedeheart registry (13) to facilitate a range of essential clinical trial procedures including recruitment, randomisation and the collection of numerous outcome measures. In additional publications reporting the follow-up data from the TASTE trial, researchers further described their trial methodology as a "registry based randomised clinical trial" $(14,15)$.

Additional noteworthy examples of research completed which mirror the rRCT methodology include 'Randomized Database Studies' (16), a publication describing the advantages of including a randomisation module within large patient databases, to strengthen the conduct of RCTs and 'Point of Care Trials' (17), which saw pragmatic RCTs using routinely collected electronic health records to ease the recruitment process and collection of trial data within studies.

While in practice, some rRCTs are described in the literature as "registry-based", their registry linked trial activities can be dissimilar, thus causing difficulty in interpreting the exact definition of an rRCT. In some cases, rRCTs do not include 'registry based' in their title even though a registry is incorporated into its protocol $(18,19)$. Examples of rRCTs that include the use of a registry within their title include The Redox Trial (20), EFFORT Trial(21) and HOPPSA trial (22). Each of these studies give clear descriptions of registry linked trial activities within their respective publications.

The CONSORT group recently published the proposed "Consort Extension for Trials Conducted in Existing Data Structures" (23). The publication of this document highlights a lack of consensus which currently exists for the reporting of randomised trials incorporating electronic data structures into their protocol, including rRCTs. This document also points to the need for clear guidance on the reporting of this trial methodology. This systematic review, focused exclusively on rRCTs, will synthesise the existing literature of published rRCTs and extract all registry linked data to define and categorise their key, critical features. The results from this study will allow replication this randomised controlled trial design with ease, and should add knowledge to the current proposed CONSORT extension, presently underway.

\section{Methods/design}

This protocol has been written in accordance with the recommendation of Preferred Reporting Items for Systematic Review and Meta-Analysis (PRISMA-P) 2015 Protocol (24). 


\section{Main Objective of the Review}

The purpose of this study is to define the key features of rRCTs by identifying RCTs that utilise a registry, establish the common registry linked trial activities in each rRCT and categorise them.

\section{Eligibility Criteria}

\section{Study designs}

Registry based randomised controlled trials, specifically utilising a patient registry (10) to facilitate key clinical trial procedures will be eligible for inclusion. This can include any clinical trial procedure or process embedded within the study protocol, clearly described within the trial publication. Both ongoing and completed studies will be eligible for inclusion. Articles published in the English language only will be included in this systematic review. No other filters will be applied (Table 1).

\section{Participants}

Studies including adult and children participants (including the parents/guardians of children) enrolled to completed or ongoing rRCTs are eligible. There is no age limit for participants.

\section{Interventions}

rRCTs investigating the treatment of any disease/pharmacological and non-pharmacological agents will be included. Randomisation within trials can be at the individual or cluster level.

\section{Comparator}

Studies will not be included or excluded from this systematic review based on comparator or control.

\section{Outcome of Interest}

Studies will not be included or excluded based on clinical outcome measures however studies must utilise a patient registry to acquire at least one study outcome measure.

\section{Table 1. Selection Criteria of Published Studies Eligible for Review}

\section{Inclusion Criteria:}


- rRCTs incorporating a patient registry to facilitate key trial procedures.

- rRCTs where randomisation is at individual or cluster level.

- rRCTs including collection of at least one outcome measure from a patient registry.

\section{Exclusion Criteria:}

- Non randomised trials.

- Trials using public health registries.

- Primary trials only - RCTs accessing registries to acquire long term follow up data will be excluded.

\section{Search Methods}

\section{Electronic Searches}

The following databases will be searched, using a combination of subject headings, MeSH and free text terms:

- MEDLINE/PubMed EBSCO

- EMBASE (OVID interface)

- CINAHLPlus EBSCO

- Scopus - Elsevier

- CENTRAL

- Cochrane Library (Cochrane Database of Systematic Reviews)

\section{Additional Searches}

The following additional sources will be searched:

- WHO International Clinical Trials Registry Platform (ICTRP): A search of the ICTRP will be conducted to extract any planned or ongoing trials.

- Reference Lists: The reference lists of included studies (snowballing-backward), citing articles (snowballing-forward) and reference lists of published systematic reviews in the field will be completed.

- Google Scholar: Google Scholar will be searched to ensure that relevant articles were not missed.

\section{Search Strategy}


The initial search be completed in PubMed and will be based on the following syntax, using the following terms:

randomised $O R$ randomized $O R$ randomized controlled trial OR randomized controlled trial (MESH) OR RCT OR "randomized clinical trial" OR pragmatic trial OR randomized database trial OR randomized registry trial OR "database study" AND Registry (MESH) OR "registry based" OR registry based OR register based OR "registry trial" OR rRCT OR register

Search terms will be adapted as appropriate for each database. The literature search and screening process will take place over six weeks. The completed search will be repeated prior to submission of the manuscript to ensure the most up to date rRCTs, if published, are retrieved and included.

\section{Study Screening}

NOS will systematically compile and import titles and abstracts of all electronic search results to Endnote, separating each database by group. Duplicate studies will be electronically removed by means of EndNote software. The remaining studies will be imported to Rayyan QCRI Software for screening (25). The screening process will involve two reviewers (NOS and FS). Both reviewers will independently review all titles, abstracts and relevant full texts for eligibility. Should discord arise during the literature screening process, the reviewers will meet to resolve any disagreement by consensus and where this is not possible a third reviewer (JE) will be consulted. All ambiguous citations will be included for full text review and where possible study authors will be contacted for clarification.

\section{Data Extraction and Management}

Following screening, all data regarding registry based trial activities will be extracted and imported to a standardized data extraction form. Data to be extracted is included in Table 2. Trial authors will be contacted where clarification is required in cases where relevant data is not available within an article. Where contact is not possible, ambiguous information as detailed in articles will be highlighted and included in the data extraction table. 
Table 2

Data Extraction Form

\begin{tabular}{|c|c|}
\hline \multirow[t]{2}{*}{ Variable } & 1. Trial Title, Author and Year \\
\hline & 2. Disease Under Investigation \\
\hline & 3. Planned Sample Size (PSS) \\
\hline & or Total Enrolled (TE) \\
\hline & 4. Registry Name \\
\hline & 5. Registry Description \\
\hline & 6. Role of Registry within Trial \\
\hline & 7. Role of Registry within Trial \\
\hline & 8. Indicators of Study Quality \\
\hline
\end{tabular}

\section{Study Quality Assessment}

NOS and FS will independently assess risk of bias for each included study, using Version 2 of the Cochrane risk-of-bias tool for randomised trials (RoB 2) (26). The risk of bias will be assessed according to the following domains: allocation concealment, blinding of participants and personnel, blinding of outcome assessment, incomplete outcome data, selective outcome reporting, similarity of baseline characteristics, and any other bias. A proposed judgement about the risk of bias arising from each domain is generated by an algorithm, based on answers to the signalling questions. Judgement can be 'Low' or 'High' risk of bias, or can express 'Some Concerns'. We will not exclude studies on the grounds of their risk of bias, but will clearly report the risk of bias when presenting the results of the studies.

\section{Data Synthesis}

A thematic analysis of the data will be conducted. This approach, known as a narrative synthesis, will be adopted based on the Guidance on the Conduct of Narrative Synthesis in Systematic Reviews (27). We will focus on the reported, documented information within each article, specifically regarding registry linked trial activities, conducting a thematic analysis of the study text, to define the key, critical features of rRCTS. We will perform a meta-analysis if appropriate, but we expect interventions will be substantially different between studies therefore it is unlikely a meta-analysis will be undertaken.

\section{Discussion}


Large, pragmatic randomised trials sit at the top of the pyramid of the hierarchy of clinical evidence. The rRCT design, while still a relatively novel undertaking, has many reported advantages to the traditional RCT endeavour. While It is essential that trialists and researchers can review published rRCTs, and attempt to duplicate trial designs as well as merge their findings in meta-analysis, there is a distinct lack of clarity in terms of the reporting of these trials. This comprehensive review of published rRCTs aims to give clarity to this trial design, defining their key, critical features, thus allowing researchers to replicate the novel rRCT trial methodology.

\section{Abbreviations}

Cinahl: Cumulative Index to Nursing and Allied Health Literature; EMBASE: Exerpta Medica Database; PRISMA: Preferred Reporting Items for Systematic Reviews and Meta-Analysis; Prospero: International Prospective Register of Systematic Reviews RCT: Randomised Controlled Trial; rRCT: Registry Based Randomised Controlled Trial;

\section{Declarations}

\section{Ethics approval and consent to participate}

Not Applicable

\section{Consent for publication}

NOS, JE and FS consent for publication.

\section{Availability of data and materials}

Data sharing is not applicable to this protocol as no data sets will be generated as part of this study.

\section{Competing interests}

The authors confirm that they have no competing interests.

\section{Funding and Acknowledgements}

This research forms part of a doctoral research project supported by the Health Research

Board Trial Methodology Research Network (HRB-TMRN) PhD scholarship awarded to NOS. The protocol represents the views of the named authors only. The funders had no role in study design, data collection and analysis, decision to publish, or preparation of the protocol. 


\section{Authors' contributions}

NOS designed and drafted the protocol and is the guarantor if this protocol; JE and FS reviewed the protocol. All authors read and approved the final manuscript.

\section{References}

1. Yndigegn $T$, Hofmann R, Jernberg T, Gale CP. Registry-based randomised clinical trial: efficient evaluation of generic pharmacotherapies in the contemporary era. Heart. 2018;104(19):1562-7.

2. Bergqvist D, Björck M, Säwe J, Troëng T. Randomized trials or population-based registries. Eur J Vasc Endovasc Surg. 2007;34(3):253-6.

3. Erlinge D, Omerovic E, Fröbert O, Linder R, Danielewicz M, Hamid M, et al. Bivalirudin versus Heparin Monotherapy in Myocardial Infarction. N Engl J Med. 2017;377(12):1132-42.

4. Götberg M, Christiansen EH, Gudmundsdottir IJ, Sandhall L, Danielewicz M, Jakobsen L, et al. Instantaneous Wave-free Ratio versus Fractional Flow Reserve to Guide PCI. N Engl J Med. 2017;376(19):1813-23.

5. Fröbert O, Lagerqvist B, Olivecrona GK, Omerovic E, Gudnason T, Maeng M, et al. Thrombus aspiration during ST-segment elevation myocardial infarction. N Engl J Med. 2013;369(17):1587-97.

6. Rao SV, Hess CN, Barham B, Aberle LH, Anstrom KJ, Patel TB, et al. A registry-based randomized trial comparing radial and femoral approaches in women undergoing percutaneous coronary intervention: the SAFE-PCI for Women (Study of Access Site for Enhancement of PCl for Women) trial. JACC Cardiovascular interventions. 2014;7(8):857-67.

7. James S, Rao SV, Granger CB. Registry-based randomized clinical trials-a new clinical trial paradigm. Nature reviews Cardiology. 2015;12(5):312-6.

8. Li G, Sajobi TT, Menon BK, Korngut L, Lowerison M, James M, et al. Registry-based randomized controlled trials- what are the advantages, challenges, and areas for future research? J Clin Epidemiol. 2016;80:16-24.

9. Methodological guidelines and. recommendations for efficient and rational governance of patient registries. 2015.

10. AHRQ Methods for Effective Health Care. In. Gliklich RE, Dreyer NA, Leavy MB, editors. Registries for Evaluating Patient Outcomes: A User's Guide. Rockville (MD): Agency for Healthcare Research and Quality (US); 2014.

11. Hoque DME, Kumari V, Hoque M, Ruseckaite R, Romero L, Evans SM. Impact of clinical registries on quality of patient care and clinical outcomes: A systematic review. PloS one. 2017;12(9):e0183667-e.

12. Frobert $O$, Lagerqvist $B$, Gudnason $T$, Thuesen L, Svensson R, Olivecrona GK, et al. Thrombus Aspiration in ST-Elevation myocardial infarction in Scandinavia (TASTE trial). A multicenter, prospective, randomized, controlled clinical registry trial based on the Swedish angiography and 
angioplasty registry (SCAAR) platform. Study design and rationale. American heart journal. 2010;160(6):1042-8.

13. Sweden's new online cardiac registry, the first of its kind. European Heart Journal. 2009. European Heart Journal. (2009) 2009.

14. Fröbert O, Lagerqvist B, Olivecrona GK, Omerovic E, Gudnason T, Maeng M, et al. Thrombus aspiration during ST-segment elevation myocardial infarction. $\mathrm{N}$ engl j med. 2013;369:1587-97.

15. Lagerqvist B, Fröbert O, Olivecrona GK, Gudnason T, Maeng M, Alström P, et al. Outcomes 1 year after thrombus aspiration for myocardial infarction. N Engl J Med. 2014;371(12):1111-20.

16. Sacristán JA, Soto J, Galende I, Hylan TR. Randomized database studies: a new method to assess drugs' effectiveness? J Clin Epidemiol. 1998;51(9):713-5.

17. van Staa TP, Dyson L, McCann G, Padmanabhan S, Belatri R, Goldacre B, et al. The opportunities and challenges of pragmatic point-of-care randomised trials using routinely collected electronic records: evaluations of two exemplar trials. Health Technol Assess (Winchester Eng). 2014;18(43):1-146.

18. Borg K, Sutton K, Beasley M, Tull F, Faulkner N, Halliday J, et al. Communication-based interventions for increasing influenza vaccination rates among Aboriginal children: A randomised controlled trial. Vaccine. 2018;36(45):6790-5.

19. Weigel S, Gerss J, Hense HW, Krischke M, Sommer A, Czwoydzinski J, et al. Digital breast tomosynthesis plus synthesised images versus standard full-field digital mammography in population-based screening (TOSYMA): protocol of a randomised controlled trial. BMJ open. 2018;8(5):e020475.

20. Sundh J, Bornefalk-Hermansson A, Ahmadi Z, Blomberg A, Janson C, Currow DC, et al. REgistrybased randomized controlled trial of treatment and Duration and mortality in long-term OXygen therapy (REDOX) study protocol. BMC pulmonary medicine. 2019;19(1):50.

21. Heyland DK, Patel J, Bear D, Sacks G, Nixdorf H, Dolan J, et al. The Effect of Higher Protein Dosing in Critically III Patients: A Multicenter Registry-Based Randomized Trial: The EFFORT Trial. JPEN Journal of parenteral enteral nutrition. 2019;43(3):326-34.

22. Idahl A, Darelius A, Sundfeldt K, Pålsson M, Strandell A. Hysterectomy and opportunistic salpingectomy (HOPPSA): study protocol for a register-based randomized controlled trial. Trials. 2019;20(1):10.

23. Kwakkenbos L, Juszczak E, Hemkens LG, Sampson M, Fröbert O, Relton C, et al. Protocol for the development of a CONSORT extension for RCTs using cohorts and routinely collected health data. Research integrity peer review. 2018;3:9.

24. Moher D, Shamseer L, Clarke M, Ghersi D, Liberati A, Petticrew M, et al. Preferred reporting items for systematic review and meta-analysis protocols (PRISMA-P) 2015 statement. Systematic reviews. 2015;4(1):1.

25. Ouzzani M, Hammady H, Fedorowicz Z, Elmagarmid A. Rayyan-a web and mobile app for systematic reviews. Systematic reviews. 2016;5(1):210. 
26. Sterne JAC, Savović J, Page MJ, Elbers RG, Blencowe NS, Boutron I, et al. RoB 2: a revised tool for assessing risk of bias in randomised trials. BMJ. 2019;366:14898.

27. Popay J, Roberts H, Sowden A, Petticrew M, Arai L, Rodgers M, et al. Guidance on the conduct of narrative synthesis in systematic reviews. A product from the ESRC methods programme Version. 2006;1:b92.

\section{Supplementary Files}

This is a list of supplementary files associated with this preprint. Click to download.

- AdditionalFile1.PRISMAPchecklist.docx 